\title{
Pourquoi faire des variétés synthétiques ?
}

\author{
A Gallais \\ INRA, station de génétique végétale, UPS, Ferme du Moulon, 91190 Gif-Sur-Yvette; \\ Institut national agronomique Paris-Grignon, 16, rue Claude-Bernard, 75231 Paris Cedex 05, France
}

(Reçu le 20 Octobre 1991; accepté le 11 juin 1992)

\begin{abstract}
Résumé - Dans cette discussion sur les variètés synthétiques, les avantages et les inconvénients connus de ce type de variétés sont rappelés. Puis la valeur des meilleures variétés synthétiques possibles à partir d'une population est comparée à la valeur des meilleures hybrides simples (ou des meilleurs clones) et à la valeur des meilleures lignées. Il est montré que les meilleurs variétés synthétiques sont toujours inférieures aux meilleurs hybrides simples et à peine supérieures (de 0 à 10\%) à la valeur de la population de départ, et d'autant moins que la dépression de consanguinité est élevée. En fait, la sélection entre variétés, ou son équivalent, compense à peine la dépression de consanguinité si celle-ci est assez forte. Si elle est faible, alors il y a intérêt à développer des variétés lignées. Le seuil dépend des variances génétiques entre lignées et entre hybrides. Dans le cas où les variétés synthétiques se justifient le plus, la population elle-même pourrait être prise comme variété sans perte importante de gain potentiel. Pour développer des variétés synthétiques, il faut donc investir surtout dans la sélection récurrente pour améliorer la valeur propre de la population et très peu dans la sélection entre variétés synthétiques à base étroite.
\end{abstract}

\section{variété synthétique / hybride / lignée / sélection récurrente}

Summary - Why develop synthetic varieties? After reviewing the known advantages and disadvantages of synthetic varieties, the value of the best possible synthetic varieties from a given population has been compared to that of the best single-cross hybrids (or the best clones) and to that of the best lines. It has been demonstrated that the best synthetic varieties are always inferior to the best single-cross hybrids and hardly superior to the original population (from 0 to 10\%), and that this inferiority is more accentuated the higher the inbreeding depression. In fact, selection between varieties or its equivalent hardly compensates for the inbreeding depression if the latter is sufficiently high. If, however, it is weak, then the development of line varieties is more justified. The threshold depends on the genetic variances among lines and among hybrids. Under conditions in which synthetic varieties are most justified, the original population could be taken as a variety without significant loss of potential gain. To develop synthetic varieties, it is therefore necessary to concentrate on recurrent selection rather than on selection between narrow-base varieties in order to improve the overall value of the population.

synthetic variety / hybrid / line / recurrent selection

\section{INTRODUCTION}

Une variété synthétique est une population artificielle dérivant de la multiplication (sexuée) pendant un nombre déterminé de générations, de la descendance du croisement naturel (synthèse) d'un certain nombre de constituants (clones, lignées, familles...), choisis pour leurs caractéristiques de valeur propre et de valeur en combinaison.

Les variétés synthétiques sont en général développées chez les espèces allogames, où le contrôle de l'hybridation à grande échelle est difficile, voire impossible. Elles sont l'équivalent de variétés populations reproductibles (aux aléas près des systèmes de maintien, car en fait, entre lots de semences d'une même variété synthétique, il y a une variation "normale", qui n'existe pas entre lots d'une variété lignée ou d'un hybride simple, et même d'un hybride double) (Gallais, 1989).

Elles permettent une certaine utilisation du phénomène d'hétérosis, mais la difficulté est bien de déterminer le nombre optimal de parents, tel qu'il soit suffisamment élevé pour limiter la dépression de consanguinité au cours des générations de multiplication, et suffisamment faible pour permettre une certaine sélection entre toutes les variétés synthétiques possibles pour 
un nombre de parents donné. En effet, plus ce nombre est élevé, plus la variance entre variétés synthétiques sera faible et, à la limite, la variété synthétique est représentée par la population sélectionnée : il n'y a plus de sélection possible entre variétés synthétiques.

Ce sont des variétés hétérogènes et plus le nombre de parents est élevé, plus l'hétérogénéité intravariétale risque d'être grande. Cette hétérogénéité est un invonvénient, pour certains caractères, si l'homogénéité est nécessaire (cas de certaines espèces potagères, comme la carotte, avant que l'on y fasse des variétés hybrides), mais elle peut être un avantage du point de vue de la stabilité des performances, surtout si les conditions de culture ne sont pas très bien définies. La contrepartie de cette hétérogénéité est que les performances dans un milieu donné ne peuvent pas être maximales. En effet, à partir de la meilleure variété synthétique, comme elle est formée d'un mélange de génotypes, il est en théorie possible d'extraire des génotypes (clones, hybrides...) plus performants qu'elle. L'utilisation de l'hétérosis ne peut donc pas être maximale. On peut alors s'attendre à des progrès génétiques moins élevés avec ce type de variétés qu'avec les autres.

Cependant, la plupart des sélectionneurs confrontés à la sélection de variétés synthétiques investissent sans doute largement autant dans le processus de création variétale que s'il s'agissait d'une variété lignée d'un hybride ou d'un clone. Tout sélectionneur investit en effet dans le test des parents : étude de la valeur propre (en clones), étude de la valeur en autofécondation, étude de la valeur en combinaison (polycross, top-cross...), dans le but de réaliser l'équivalent d'une sélection entre variétés synthétiques. Les meilleurs "constituants", selon certains critères, sont sélectionnés, et intercroisés entre eux en nombre limité pour initier une ou plusieurs variétés synthétiques qui, après multiplication, seront étudiées pour leurs performances. Or, par rapport à des variétés clones, lignées ou hybrides simples, la variance entre variétés synthétiques est très faible (voir cidessous), même pour un nombre de parents assez faible (4-5). L'efficacité de l'étape création variétale sera donc faible, et d'autant plus qu'il ne suffit pas de considérer la variance entre variétés, mais aussi la moyenne de toutes les variétés. Ainsi, si la diminution du nombre de parents permet d'augmenter la variance entre variétés, elle entraîne aussi une dépression de consanguinité plus grande qui tendra à annuler l'effet de la sélection. On peut donc s'interroger sur la valorisation des investissements faits au niveau de la création de variétés synthétiques.

\section{Démarche de l'étude. Hypothèses}

À partir des expressions déjà établies (Gallais, 1989) des moyennes et des variances des différents types de variétés (lignées, clones, hybrides simples, variétés synthétiques) le but de cet article est de préciser dans quelles situations génétiques les variétés synthétiques sont justifiées par rapport aux lignées et aux hybrides simples, et quelle peut être l'importance du gain espéré. Pour cela, à partir d'une même population d'amélioration, avec l'hypothèse d'équilibre panmictique au niveau de la génération considérée (cela exclut l'autofécondation préférentielle mais non l'autostérilité), la valeur attendue des meilleures variétés synthétiques sera d'abord comparée aux meilleurs hybrides simples, puis aux meilleures lignées. Pour simplifier les comparaisons, il sera supposé que les effets de compétition sont faibles ou tels que, à une densité donnée, ils ne remettent pas en cause des relations entre moyennes ou variances qui résultent du modèle de la génétique quantitative; enfin, les parents d'une variété synthétique seront considérés non consanguins et non apparentés. En remarquant qu'avec équilibre de liaison (ou en l'absence d'épistasie) la distribution des valeurs génétiques des clones non consanguins est identique à la distribution des valeurs génétiques des hybrides simples, la comparaison des variétés synthétiques avec les hybrides simples renseignera directement sur leur valeur par rapport aux clones.

\section{Expression des moyennes et des variances des différents types de variétés}

Le tableau I montre l'expression de la moyenne et de la variance de la distribution des variétés d'un type donné : clone, hybride simple, lignée, variété synthétique à $k$ parents (Gallais, 1989). Dans le cas d'additivité stricte, le plus simple à considérer, l'expression de la variance entre variétés ne dépend que de la variance d'additivité $\left(\sigma_{A}^{2}\right)$ et la variance entre variétés synthétiques est $\sigma_{\mathrm{A}}^{2} / k$. Dans le cas de dominance, l'expression de la variance entre variétés d'un type donné est un peu plus complexe. La variance entre clones est toujours égale à la variance entre hybrides 
Tableau I. Expression de la moyenne et de la variance de la distribution des variétés d'un type donné (en l'absence d'épistasie et avec l'hypothèse d'équilibre panmictique au niveau de la population d'amélioration et au niveau de la génération étudiée des variétés synthétiques.

Type de variétés

Clones

Lignées

Hybrides simples

Hybrides doubles

Variétés synthétiques

(avec clones non apparentés

et non consanguins)

Avec des lignées
Moyenne

Additivité stricte
Additivité + dominance

$\begin{array}{llc}\mu & \sigma_{A}^{2} & \sigma_{A}^{2}+\sigma_{D}^{2} \\ \mu_{L}=\mu(1-\Delta) & 2 \sigma_{A}^{2} & 2 \sigma_{A L}^{2} \\ \mu & \sigma_{A}^{2} & \sigma_{A}^{2}+\sigma_{D}^{2} \\ \mu & 1 / 2 \sigma_{A}^{2} & 1 / 2 \sigma_{A}^{2}+1 / 4 \sigma_{D}^{2} \\ \mu-1 / 2 k\left(\mu-\mu_{L}\right) & 1 / k \sigma_{A}^{2} & 1 / k \sigma_{A}^{2}+(2 k-1) / 4 k^{3} \sigma_{D}^{2} \\ =\mu(1-\Delta / 2 k) & & \\ \mu-1 / k\left(\mu-\mu_{L}\right) & 2 / k \sigma_{A}^{2} & 2 / k \sigma_{A}^{2}+2(k-1) / k^{3} \sigma_{D}^{2} \\ =\mu(1-\Delta / k) & & \end{array}$

$\mu$ : moyenne de la population d'amélioration; $\mu_{\mathrm{L}}$ : moyenne de toutes les lignées dérivables de cette population; $\Delta$ dépression maximale de consanguinité (différence relative entre hybrides et lignées, par rapport aux hybrides); $k$ : nombre de constituants (clones) de la variété synthétiques; $\sigma_{\mathrm{A}}^{2}$ : variance d'additivité; $\sigma_{\mathrm{AS}}^{2}$ : variance des effets additifs en synthèse; $\sigma_{\mathrm{D}}^{2}$ : variance de dominance.

simples de lignées; la présence de dominance fait que la variance entre hybrides simples peut approcher la variance entre lignées, mais elle n'ajoute que très peu à la variance entre variétés synthétiques. En effet, dans une variété synthétique, les effets de dominance sont beaucoup plus dilués que les effets d'additivité. Cela augmente donc encore la différence de variance entre variétés en passant du niveau hybride au niveau variété synthétique. Ainsi avec 4 parents la variance entre variétés synthétiques est au moins 4 fois plus faible que la variance entre hybrides simples. La figure 1, d'après les résultats de Gallais (1990b) montre dans le cas de biallélisme comment varie la variance entre variétés en fonction de la fréquence des gènes (avec $k=4$ pour les variétés synthétiques). Elle montre en particulier que dès que le degré de dominance augmente, le rapport variance entre hybrides/ variance entre variétés synthétiques augmente.

\section{COMPARAISON DES MEILLEURES VARIÉTÉS SYNTHÉTIQUES AUX MEILLEURS HYBRIDES SIMPLES}

La figure 2 illustre les distributions des hybrides simples et des variétés synthétiques à 4 parents, avec un rapport de 4 entre les 2 variances. Elle permet de montrer que, du fait de la nettement plus faible variance entre variétés synthétiques, il sera très difficile ou improbable, voire impossible, même avec des investissements élevés, d'isoler une variété synthétique aussi bonne que les meilleurs hybrides simples. Mais en fait, la situation est encore pire si l'on tient compte du fait que, par l'existence de la dominance, ou plus généralement de l'hétérosis, la moyenne des variétés synthétiques sera inférieure à la moyenne des hybrides simples.

Pour préciser la situation, il faut pouvoir comparer les moyennes et les variances, donc les moyennes et les écarts types des distributions. Cela peut se faire par l'utilisation des coefficients de variation. En fait, il faut comparer la valeur attendue des meilleures variétés synthétiques (Max Syn) à la valeur attendue des meilleurs hybrides (ou clones) (Max HS) :

$$
\begin{aligned}
& \operatorname{Max}(\text { Syn })=\mu_{S y n}+i_{S} \sigma_{S y n} \\
& \operatorname{Max}(H S)=\mu_{H S}+i_{H S} \sigma_{H S}
\end{aligned}
$$

où $\mu_{S y n}, \sigma_{S y n}$ (respectivement $\mu_{H S}, \sigma_{H S}$ ) représentent la moyenne et la variance de toutes les variétés synthétiques pour un nombre de parents fixé (ou de tous les hybrides simples); is (iHS) est l'intensité de sélection (ou équivalent) entre variétés synthétiques ou hybrides. Pour simplifier, le raisonnement est fait au niveau de la valeur génétique des variétés (il faudrait, en fait, tenir compte de l'héritabilité qui ne serait pas la même aux deux niveaux). 

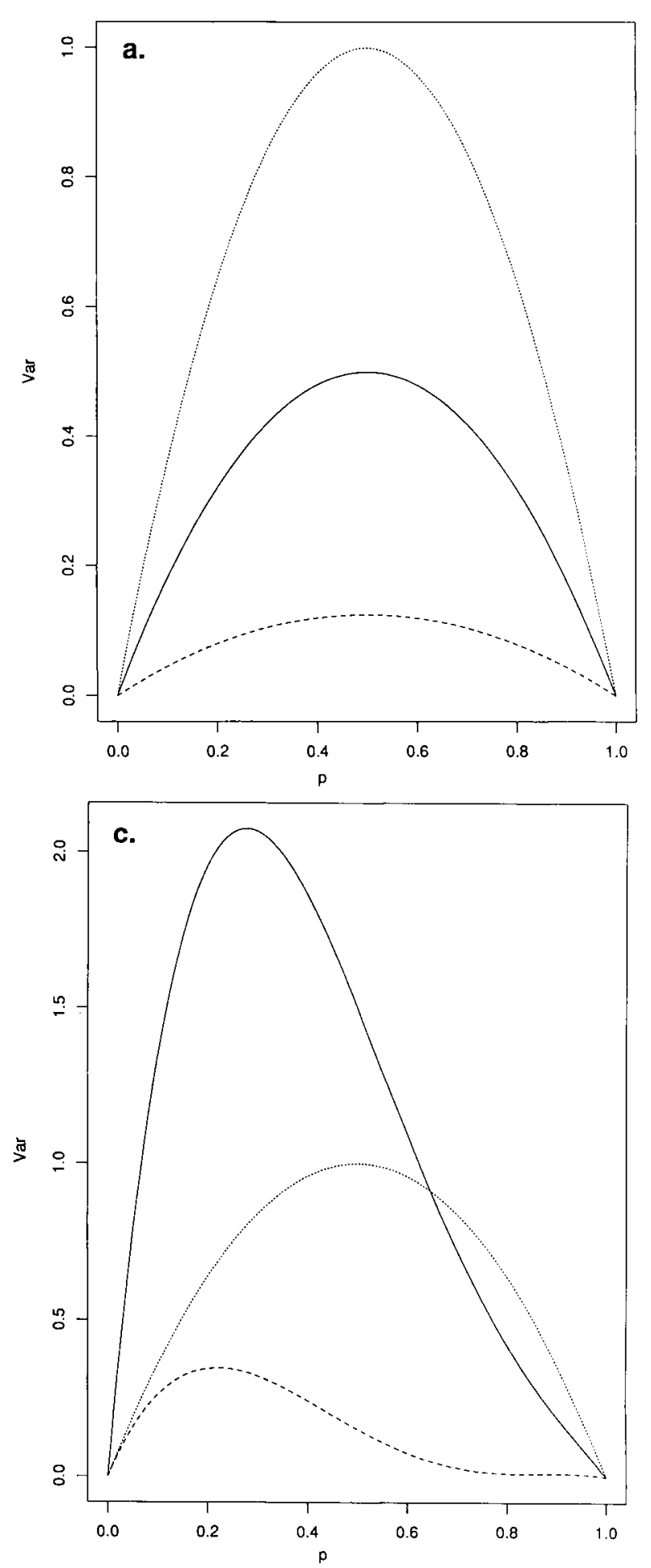

À partir de ces expressions très simples, on a donc, en supposant une même intensité de sélection pour hybrides et variétés synthétiques :

$$
\begin{aligned}
& \operatorname{Max}(H S)-\operatorname{Max}(S y n) \\
& =\left(\mu_{H S}-\mu_{S y n}\right)+i\left(\sigma_{H S}-\sigma_{S y n}\right)
\end{aligned}
$$

ou en valeur relative

$\operatorname{Max}(H S)-\operatorname{Max}(S y n)] / \mu_{H S}=(1-u)+i c(1-s)$

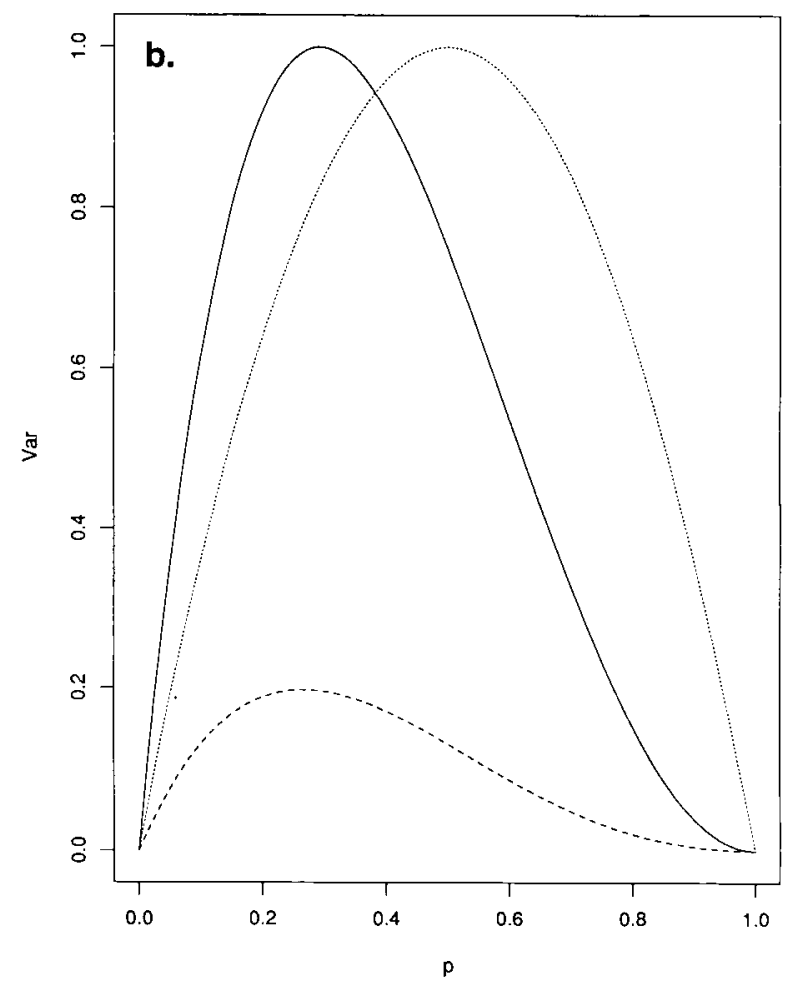

Fig 1. Évolution de la variance entre variétés dans le cas de biallélisme, pour un locus et pour différents degrés de dominance $d / a$ (en trait continu, variance entre clones ou entre hybrides simples; en pointillé, variance entre lignées; en trait discontinu, variance entre variétés synthétiques à 4 constituants non consanguins). a. Additivité, $d / a=0$. b. Dominance complète, $d / a=1$. c. Superdominance, $d / a=2$.

où 1 - $u$ représente la différence moyenne relative entre hybrides et variétés synthétiques, $c$ le coefficient de variation des hybrides, et $s$ le rapport des écart types $\sigma_{\mathrm{Syn}} / \sigma_{\mathrm{HS}}$.

La valeur de $u$ est directement liée à la dépression de consanguinité (mesurée par la différence $\Delta_{S}$ entre croisement et autofécondation) (Gallais, 1989, 1990a) :

$$
1-u=\Delta_{S} / k
$$




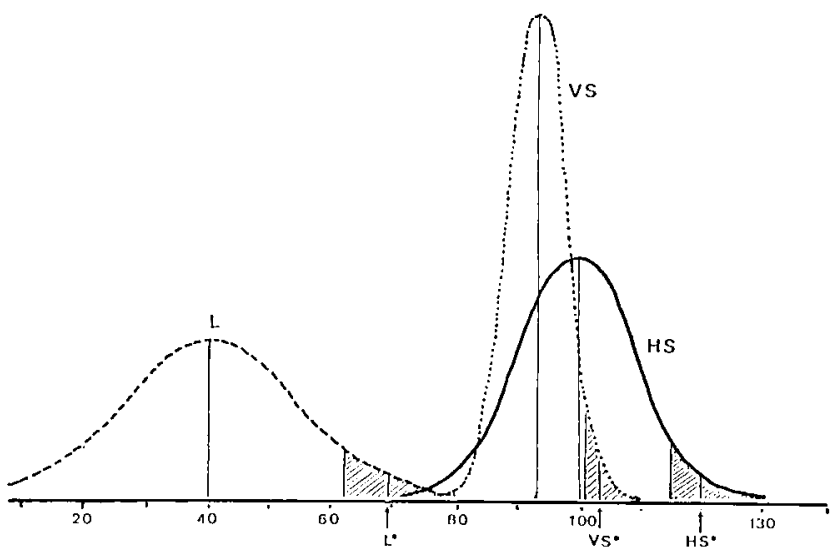

Fig 2. Comparaison de la distribution des variétés synthétiques (VS) et des hybrides (HS) $\Delta_{S}=0,30 ; c=0,10 ; k=4$; $\sigma_{\mathrm{Syn}} / \sigma_{\mathrm{HS}}=0,50$. $\dot{A}$ titre de comparaison, la distribution des lignées (L) a été placée avec un coefficient de variation égal à 0,375 . Les zones hachurées correspondent à une intensité de sélection de $2 . L^{*}, V S^{\star}, H S^{\star}$ représentent la moyenne des meilleures variétés du type considéré, pour une même intensité de sélection.

II est alors possible de situer l'écart entre hybrides et variétés synthétiques. La valeur de $\Delta_{S}$ peut varier de $0 \%$ (cas d'additivité) à $30-40 \%$ pour des espèces très sensibles à la consanguinité; une intensité de sélection "raisonnable" semble correspondre à un taux de sélection de $5 \%$ d'où $i$ $\approx 2$ pour simplifier; le coefficient de variation entre hybrides peut être compris, d'après des résultats expérimentaux (Hallauer et Miranda, 1981), entre 0,06 et 0,15 ; le rapport des écarts types $s$ dépend du nombre de constituants; avec $k=4, s \leq 1 / 2$. D'où, avec $k=4$ et pour $\Delta_{S}=0,30, i=2, c=0,10$, $s=0,50$, une différence de $17,5 \%$ entre hybrides simples et variétés synthétiques, ce qui est très important ! Mais, ce qu'il faut de plus remarquer, c'est que dans ce cas, les meilleures variétés synthétiques ne seront que de $2,5 \%$ supérieures à la valeur de la population d'amélioration d'où sont tirés les constituants (dans le cas général, l'écart entre la moyenne des meilleures variétés synthétiques et la moyenne de la population vaut en effet, en valeur relative : $i c s-\Delta_{\mathcal{S}} k$, dans l'exemple pris $\Delta \mathcal{S} k=0,075$ et $i c s=0,10$, la sélection ne fait que compenser la dépression de consanguinité). En fait, il ne faut pas une trop forte dépression de consanguinité pour avoir des variétés synthétiques supérieures à la moyenne de la population d'amélioration. Ainsi, avec $\Delta_{S}=0,15$, les "meilleures" variétés synthétiques à 4 parents seraient de $6,25 \%$ supérieures à la population d'amélioration.

L'écart entre hybrides et variétés synthétiques est bien sûr plus faible si l'on considère des hy- brides doubles; avec une variance de dominance égale à la variance d'additivité, et comme précédemment $\Delta_{S}=0,30, i=2, s=0,50, c=$ 0,10 , l'écart entre hybrides doubles et variétés synthétiques n'est alors que de $10 \%$.

Des calculs plus précis tenant compte des effets du milieu, de l'intensité de sélection qui varie selon le nombre de parents (et qui sera plus faible pour les synthétiques que pour les hybrides) (Gallais, 1990a) confirment bien que la sélection entre variétés synthétiques, ou son équivalent, ne permet pas de progresser de façon significative par rapport à la population de départ, dès que la dépression de consanguinité est importante... Or c'est souvent dans ce cas-là que des variétés synthétiques sont développées. $\mathrm{Si}$ la dépression de consanguinité est faible, il vaut alors mieux développer des lignées, si la biologie florale le permet (voir cidessous).

Ainsi, si la création de variétés synthétiques s'impose avec une dépression de consanguinité assez forte, il vaut mieux n'investir que dans l'amélioration des populations par sélection récurrente pour améliorer la valeur propre de ces populations. C'est la population améliorée ellemême qui pourra être prise comme variété. Les plantes intercroisées pour passer à la génération suivante pourront être prises comme parents de la variété synthétique, avec une intensité de sélection seulement un peu plus forte; ainsi, si à chaque cycle 30 à 40 plantes sont sélectionnées et intercroisées pour la sélection récurrente, 10 à 20 (les moins apparentées possibles) pourront être prises pour former une variété synthétique, sans autres tests que ceux réalisés pour la sélection récurrente (si le système de test est bien choisi : aptitude générale à la combinaison ou aptitude générale à la synthèse).

La restriction du nombre de parents n'est intéressante que pour progresser très rapidement sur certains caractères génétiquement simples (maladies, précocité...). Mais elle demande des investissements importants pour "éliminer" les effets néfastes de la consanguinité sur les caractères de vigueur. II faut en effet "sélectionner» entre variétés synthétiques pour "compenser» et si possible "surcompenser» les effets de la consanguinité, mais avec un progrès qui sera toujours très faible sur les caractères les plus complexes. Le faible progrès réalisé chez les plantes fourragères pérennes (luzerne, graminées) par rapport à des espèces comme le 
blé ou le maïs s'explique en partie par le fait que la sélection entre variétés ne peut pas y être aussi efficace. (L'autre cause est bien sûr la plus faible héritabilité des caractères due à de forts effets milieux).

\section{COMPARAISON DES MEILLEURES VARIÉTÉS SYNTHÉTIQUES AUX MEILLEURES LIGNÉES}

II semble assez immédiat que si les meilleures variétés synthétiques s'obtiennent lorsque la dépression de consanguinité est faible, alors dans ce cas comme la variance entre lignées est beaucoup plus forte que la variance entre variétés synthétiques, il vaudra mieux faire des lignées. Et là, il n'y a pas le même problème que pour les hybrides : s'ils sont théoriquement justifiés, encore faut-il pouvoir les produire, c'est-àdire contrôler l'hybridation à grande échelle. Pour produire des lignées, à grande échelle, chez une plante allogame, il suffit que l'autofécondation soit réalisable, avec une fertilité suffisante. Ce sera sans doute le cas si la dépression de consanguinité est suffisamment faible : cela peut signifier que l'allogamie ne présente pas un avantage très important et aucun système très efficace n'aura été mis en place pour éviter la reproduction en consanguinité. On peut citer ici le colza comme exemple, espèce pour laquelle des variétés lignées ont été développées en France, des variétés synthétiques en Europe du Nord... et maintenant il y aurait un intérêt pour les variétés hybrides.

En reprenant le raisonnement précédent, toujours au niveau des valeurs génétiques, la différence entre les meilleures variétés synthétiques (Max Syn) et les meilleures lignées (Max $L)$ peut s'écrire :

$$
\begin{aligned}
& \operatorname{Max}(\operatorname{Syn})-\operatorname{Max}(L)=\left(\mu_{\text {Syn }}-\mu_{L}\right) \\
& +i\left(\sigma_{\text {Syn }}-\sigma_{\mathrm{L}}\right)
\end{aligned}
$$

$\mu_{\mathrm{L}}$ étant la moyenne et $\sigma_{\mathrm{L}}^{2}$ la variance de toutes les lignées dérivables de la population d'amélioration. D'après les expressions de $\mu_{\mathrm{L}}$ et de $\mu_{\mathrm{Syn}}$ données dans le tableau I, cette expression, en valeur relative par rapport à la moyenne des lignées, peut encore s'écrire :

$$
[\Delta /(1-\Delta)](1-1 / 2 k)-i C_{L}(1-v)
$$

ou par rapport à la moyenne des hybrides:

$$
\Delta(1-1 / 2 k)-i(1-\Delta) c_{\mathrm{L}}(1-v)
$$

$\Delta$ représentant la dépression relative totale de consanguinité (différence relative entre hybrides et lignées exprimée par rapport aux hybrides; en l'absence d'épistasie $\Delta=2 \Delta_{S}$ ); $q_{\mathrm{L}}$ est le coefficient de variation entre lignées et $v$ est le rapport des écarts types $\sigma_{S y n} / \sigma_{L}$.

Le premier terme de la différence, toujours positif, exprime le fait que la moyenne des variétés synthétiques est supérieure à la moyenne des lignées, mais le deuxième terme se retranche, du fait que la variance entre variétés synthétiques est attendue inférieure à la variance entre lignées $(v<1)$. Pour préciser le signe et l'ampleur de cette différence, il faut donner des valeurs aux paramètres $\Delta, i, c_{L}, v . \Delta$ peut être pris voisin de $0,20-0,30$, (dépression moyenne), $i$ comme précédemment sera pris égal à 2 pour simplifier, bien que l'intensité de sélection entre variétés synthétiques soit plus faible que l'intensité de sélection entre lignées; $c_{\mathrm{L}}$, le coefficient de variation génétique entre lignées, est attendu supérieur à celui des hybrides; des valeurs comprises entre 0,20 et 0,30 semblent probables (d'après des résultats expérimentaux sur blé et sur maïs). Si l'on se donne les paramètres $s=\sigma_{\mathrm{Syn}} / \sigma_{\mathrm{HS}}$, $c, c_{\mathrm{L}}$ et $\Delta$, il en résulte :

$$
v=s c /\left[c_{\mathrm{L}}(1-\Delta)\right]
$$

Avec $\Delta=0,25, k=4, i=2, c_{L}=0,20$ par exemple les distributions correspondantes sont représentées sur la figure 3 et la différence entre les meilleures variétés synthétiques et les meilleures lignées n'est que de 2,7\%; cette valeur est d'ailleurs une surestimation de ce qui serait plus réel en considérant une intensité de sélection

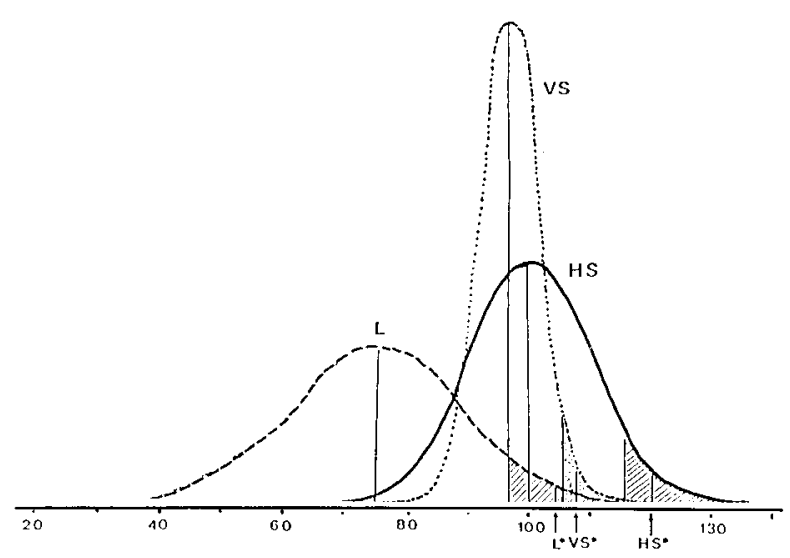

Fig 3. Comparaison de la distribution des variétés synthétiques (VS) et des lignées (L). $\Delta=0,25 ; k=4 ; c_{\mathrm{L}}=0,20 ; \sigma_{\mathrm{Syn}}$ ' $\sigma_{L}: 0,333$. Avec ce jeu de paramètre, les valeurs des meilleures variétés synthétiques et des meilleures lignées sont très proches. À titre de comparaison, la distribution des hybrides simples (HS) a été placée, avec un coefficient de variation égal à 0,10 . 
entre variétés synthétiques plus faible que celle entre lignées. Si le coefficient de variation entre lignées est plus élevé ou si la dépression de consanguinité est plus faible, il sera plus justifié de développer des lignées; par contre si la dépression de consanguinité est plus forte il vaudra mieux développer des variétés synthétiques selon la méthode exposée précédemment, c'està-dire comme coproduit de la sélection récurrente sur la valeur en synthèse.

\section{DISCUSSION ET CONCLUSION}

Si aux deux comparaisons précédentes on ajoute la comparaison entre hybrides simples et lignées déjà discutée par Gallais (1989), alors il est possible de déterminer les domaines pour lesquels tel type de variétés est plus justifié qu'un autre. Pour représenter ces domaines (fig $4 a$ et $4 b$ ), l'intensité de sélection a été fixée à 2 et le nombre des constituants des variétés synthétiques a été choisi à 4 . À partir de valeurs réalistes, la figure $4 \mathrm{a}$ définit les domaines en fonction des paramètres $\Delta$ et $c$, les autres paramètres étant fixés, et la figure $4 b$ définit les domaines en fonction des paramètres $\Delta$ et $\sigma_{L} / \sigma_{H S}$, les autres paramètres étant fixés.

La comparaison des meilleures variétés synthétiques aux meilleurs hybrides simples (ou clones) et aux meilleures lignées, à partir d'une même population de départ, montre qu'il n'y a pas de situation sur le plan génétique, du point

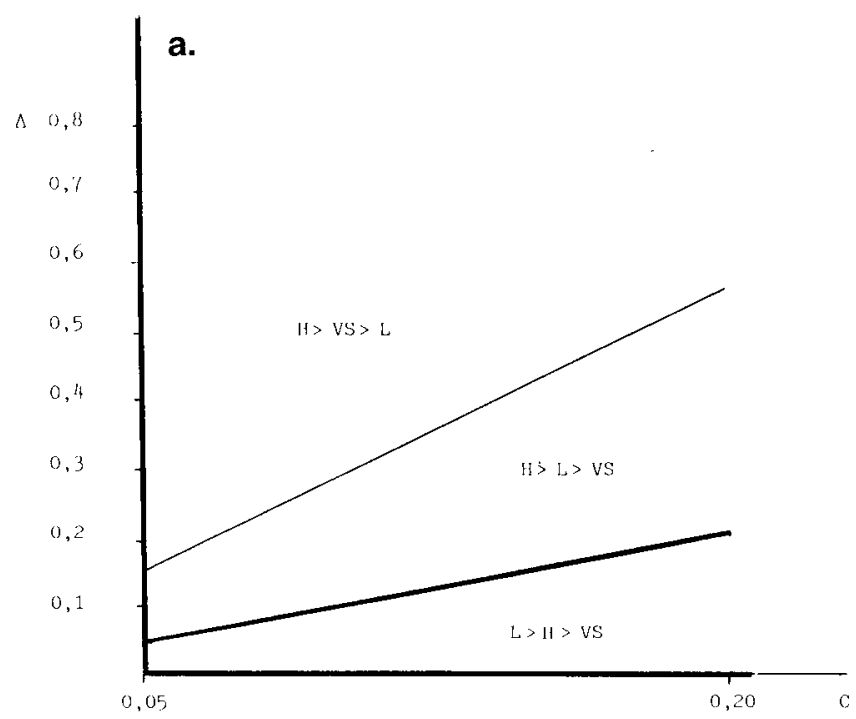

de vue des performances maximales, où il soit justifié de développer des variétés synthétiques. Si la dépression de consanguinité est importante, il faudrait développer des hybrides, mais le problème est de contrôler l'hybridation à grande échelle; si la dépression de consanguinité est plus modérée, il est plus justifié de développer des lignées. Les variétés synthétiques ne sont justifiées que si la dépression de consanguinité est assez importante et si le contrôle de l'hybridation est impossible à grande échelle. De toute façon, c'est un type de variétés «provisoire», en attendant la maîtrise de l'hybridation à grande échelle ou la mise au point de graines artificielles. Cette conclusion n'est pas très nouvelle, mais il est important de bien la souligner au sélectionneur qui n'aurait pas conscience de l'écart entre le meilleur hybride simple et la meilleur variété synthétique, et qui tendrait peut-être à faire des variétés synthétiques par «tradition».

Pour les espèces mixtes semi-allogames (comme le colza) avec une dépression de consanguinité assez modérée, il est clair que si le contrôle de l'hybridation est impossible à grande échelle, il vaut mieux faire des lignées plutôt que des variétés synthétiques, en attendant de pouvoir développer des hybrides. Les figures $4 a$ et $4 b$ montrent que le seuil de dépression de consanguinité qui justifie des lignées plutôt que des variétés synthétiques dépend de la variation génétique : il augmente lorsque la variation génétique augmente et lorsque la variation des lignées croît par rapport à celle des hybrides.

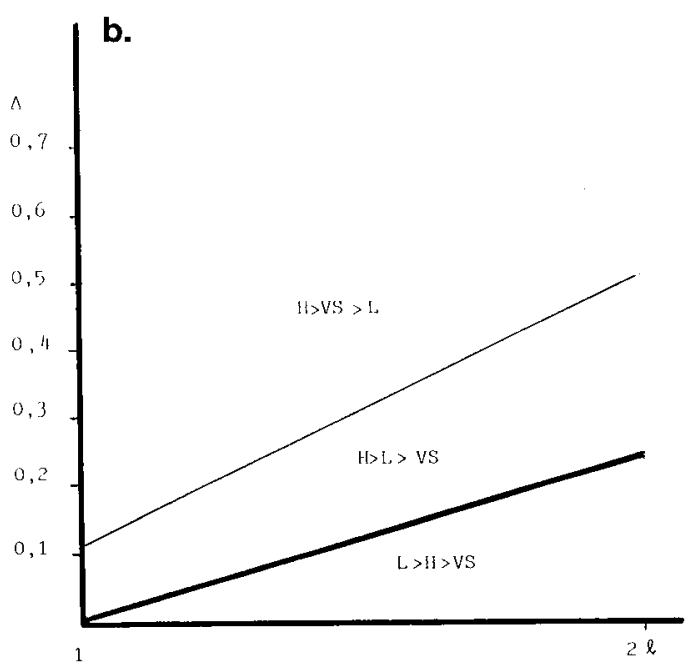

Fig 4. Illustration des différents domaines possibles du point de vue du classement des 3 types de variétés hybrides $(H)$, variétés synthétiques (VS) et lignées (L). H est toujours supérieur à VS car $\Delta 2 k>i c(s-1)$; VS $>L$ si $\Delta(1-1 / 2 k) i c(I-s) ; H>L$ si $\Delta>i$ $c(l-1)$. a. Avec $\mathrm{l}$, racine du rapport de la variance entre lignées à la variance entre hybrides, domaines $\mathrm{f}(\Delta, i)$, les autres paramètres étant fixés. Pour la représentation ci-dessus, $k=4, i=2, l=1,5$ et $s=0,5$. b. Domaines $f(\Delta, h)$, les autres paramètres étant fixés; $k=$ $4, i=2, c=0,10, s=0,5$. 
L'existence d'interactions entre plantes va-t-elle modifier les conclusions ? À une densité donnée, la compétition va augmenter le nombre de paramètres nécessaires pour décrire les moyennes et les variances des différents types de variétés (Gallais, 1975b, 1976). Ainsi, au niveau des variétés synthétiques, cela pourra se traduire par une dépression de consanguinité plus faible qu'attendue sur la base de la différence entre la moyenne des hybrides et celle des lignées, non seulement à cause des phénomènes de compensation et de domination des plantes les plus vigoureuses dans un peuplement hétérogène, mais aussi par l'élimination des plantes les plus consanguines. Un tel mécanisme tendra aussi à diminuer la variance entre variétés synthétiques à faible nombre de constituants par rapport à celle prévue sur la base d'un test séparé de l'aptitude générale à la combinaison (AGC) et de la valeur en autofécondation des constituants. Pour des nombres de parents plus élevés $(>4)$, la variance entre variétés synthétiques peut toujours se prévoir à partir de la variance d'AGC appréciée en compétition. En effet, des travaux de Gallais (1975b) et de Rotili et Zannone (1975) montrent que les tests de descendances demi-frères en culture pure ou en mélange donnent sensiblement les mêmes résultats.

Avec l'estimation directe des moyennes et des variances des différents types de variétés en conditions de compétition, la démarche réalisée dans cette étude peut toujours être développée, quelle que soit la situation biologique (compétition, autofertilité...). Quantitativement et qualitativement pour de nombreuses situations, les conclusions précédentes restent valables. Ainsi, quelles que soient les interactions entre plantes et avec une dépression de consanguinité seulement moyenne, les meilleures variétés synthétiques possibles ne seront jamais très supérieures à la population d'amélioration. L'avantage des hybrides par rapport à cette population dépendra surtout de la variance entre hybrides.

La conclusion la plus importante qui émerge de cette discussion est bien qu'il n'y a pratiquement rien à gagner par rapport à la moyenne de la population panmictique par une sélection entre variétés synthétiques avec un nombre réduit de parents. Le gain par rapport à la population sera au plus de $10-15 \%$ avec une dépression de consanguinité moyenne, et souvent moins (0 à $5 \%$ ) dès que la dépression de consanguinité sera assez importante. Comme, en augmentant le nombre de parents, l'effet de la sélection devient très faible, la meilleure stratégie est d'améliorer par sélection récurrente la valeur propre de la population et d'utiliser celle-ci comme variété synthétique, les parents étant les plantes retenues pour l'intercroisement.

La seule justification de la restriction de la base génétique est non pas d'essayer de progresser sur un caractère complexe comme le rendement, mais plutôt sur des caractères plus simples (oligogéniques) comme la préco-cité, les résistances aux maladies, et pour lesquels il vaut mieux une variété homogène. Le problème est que cette restriction de la base génétique impose des tests de rendement : les parents pourront être choisis sur leur aptitude générale à la combinaison (AGC) (mesurée en top cross) ou mieux pour une base très étroite, sur un index de leur AGC et de leur valeur S1, c'est-à-dire sur leur aptitude générale à la synthèse (Gallais, 1975a, 1979; Wright, 1974). Mais ces tests sont coûteux pour un progrès faible ou nul sur le rendement (voire même une perte!). La meilleure stratégie est donc bien d'utiliser la population améliorée comme variété synthétique.

Cette stratégie présente bien des avantages: elle diminue le coût de la sélection des variétés synthétiques et de plus elle permet sans doute de créer des variétés plus stables de comportement selon le milieu (par leur hétérogénéité). Le progrès sur le rendement ne sera pas maximal, mais sera de toute façon assez proche du progrès optimal. Le faible gain attendu (quelques pourcent) par la restriction de la base génétique étant extrêmement coûteux pour le sélectionneur, il vaut sans doute mieux qu'il consacre le maximum de moyens à la sélection récurrente pour augmenter l'efficacité de celle-ci. De plus, si elle est bien conduite en améliorant simultanément la valeur en autofécondation et l'aptitude à la combinaison (les composantes de la valeur en synthèse), elle peut préparer le développement de variétés hybrides simples qui nécessite des lignées à la fois de bonne valeur propre et de bonne valeur en combinaison.

\section{RÉFÉRENCES}

Gallais A (1975a) Prévision de la vigueur et sélection des parents d'une variété synthétique. Ann Amélior Plant 25, 233-264

Gallais A (1975b) Sur quelques aspects de la compétition en amélioration des plantes. Ann Amélior Plant $25,233-264$ 
Gallais A (1976) Effects of competition on means, variances and covariances in quantitative genetics, with an application to general combining ability selection. Theor Appl Genet 47, 189-195

Gallais A (1979) Application du concept d'aptitude variétale à la théorie de la sélection des variétés synthétiques chez les diploïdes et les autopolyploïdes. Ann Amélior Plant 29, 111-129

Gallais A (1989) Théorie de la sélection en amélioration des plantes. Masson, Paris, $588 \mathrm{p}$

Gallais A (1990a) Theoretical determination of the optimum number of parents for synthetics. Theor Appl Genet 79, 417-421
Gallais A (1990b) Concepts of varietal value and of test value. Application to recurrent selection. A synthesis. Euphytica 48, 197-209

Hallauer AR, Miranda JB (1981) Quantitative genetics in maize breeding. lowa State University Press, Ames, IA, $468 p$

Rotili $P$, Zannone L (1975) Les principaux aspects d'un méthode de sélection de la luzerne basée sur les dispositifs qui utilisent la concurrence entre plantes. Ann Amélior Plant 25, 29-49

Wright AJ (1974) A genetic theory of general varietal ability for diploid crops. Theor Appl Genet 45, 163-169 